\title{
TOP2 gene disruption reduces drug susceptibility by increasing intracellular ergosterol biosynthesis in Candida albicans
}

Correspondence

Hui Ji

huijicpu@163.com

Yong-Bing Cao

lengao1228@163.com

Received 16 December 2009

Accepted 9 March 2010

\author{
Hao Zheng, ${ }^{1,2}$ Yuan-Ying Jiang, ${ }^{2}$ Yan Wang, ${ }^{2}$ Xin-Ming Jia, ${ }^{2}$ \\ Tian-Hua Yan, ${ }^{1}$ Ping-Hui Gao, ${ }^{2}$ Lan Yan, ${ }^{2}$ Ling-Huo Jiang, ${ }^{3}$ Hui $\mathrm{Ji}^{1}$ \\ and Yong-Bing $\mathrm{CaO}^{2}$
}

\author{
${ }^{1}$ Department of Pharmacology, China Pharmaceutical University, Nanjing, PR China \\ ${ }^{2}$ School of Pharmacy, Second Military Medical University, Shanghai, PR China \\ ${ }^{3}$ School of Pharmaceutical Science and Technology, Tianjin University, PR China
}

\begin{abstract}
In this study the role of the TOP2 gene in fungal drug susceptibility was investigated by disrupting and overexpressing the gene in Candida albicans. MIC determination and a spot assay showed that a top $2 \Delta / \Delta$ null mutant (strain T2bc) was more resistant to the antifungals tested than the wildtype (strain CAI4). Real-time RT-PCR and rhodamine $6 \mathrm{G}$ efflux examination showed that TOP2 did not influence the activity of drug efflux pumps. Sterol analysis with $\mathrm{GC} /$ high-resolution MS indicated that the intracellular ergosterol composition of the top $2 \Delta / \Delta$ mutant was significantly increased. Subsequently, fluorescence polarization measurements also revealed that Top2deprived cells displayed a decrease in membrane fluidity, resulting in enhanced passive diffusion of the drugs. Quantitative real-time RT-PCR analysis further confirmed that the ERG11 gene, an essential gene in ergosterol biosynthesis, was upregulated. These results demonstrate a close relationship between the TOP2 gene and drug susceptibility in C. albicans.
\end{abstract}

\section{INTRODUCTION}

Candida albicans is reported to be responsible for approximately $50 \%$ of all clinical fungal infections (Pappas et al., 2003; Wisplinghoff et al., 2004) and has become a prevalent opportunistic fungal pathogen (Silver et al., 2004; White et al., 2002). Following the long-term administration of the current antifungal drugs, C. albicans has developed resistance to these drugs, in particular to fluconazole (Perea et al., 2001; Dassanayake et al., 2002).

It is generally accepted that the most common mechanism underlying antifungal drug resistance is constitutive overproduction of multidrug transporters leading to drug efflux from the cells (Anderson, 2005; Shao et al., 2007; White et al., 2002). Two types of azole transporters have been defined: ATP-binding cassette transporters encoded by $C D R 1$ and $C D R 2$, and the major facilitator superfamily transporters encoded by MDR1 (Ghannoum \& Rice, 1999). Less commonly, stable resistance can also result from alterations of lanosterol $14 \alpha$-demethylase (CYP51), targeted by fluconazole, and encoded by ERG11 (Albertson et al., 1996; Perea \& Patterson, 2002). In recent years, there

Abbreviation: HRMS, high-resolution MS.

Additional figures and tables are available as supplementary material with the online version of this paper. has been documented evidence about the molecular basis of related genes in resistant strains and the transcription regulators that mediate their constitutive overexpression in drug resistance (Akins, 2005; Morschhauser, 2002).

Although the molecular basis of drug resistance in $C$. albicans is not clear, accumulated evidence indicates that multidrug resistance is a multifactorial phenomenon. Recent evidence also suggests that lipids could play an important role in drug susceptibility. Researchers have shown that some resistant $C$. albicans isolates exhibit changes in sterol composition and membrane phospholipids (Loffler et al., 2000; Kohli et al., 2002). It appears that the associated alterations in membrane lipid composition and its order (fluidity) could be significant determinants of drug susceptibility in yeast cells (Mukhopadhyay et al., 2002).

Investigations in both prokaryotes and eukaryotes have demonstrated that topoisomerase II (Top2), a DNA unwinding enzyme, has a wide spectrum of functions in cellular processes, and the ensemble of topoisomerase II enzymes in mammalian cells appears to be more complex than previously thought (Wang, 2002; Zini et al., 1992). The C. albicans TOP2 gene has been analysed by heterologous expression in Saccharomyces cerevisiae to study Top2 inhibitors (Keller et al., 1997). Recent studies in our 
laboratory have indicated that there may be a relationship between the TOP2 gene and drug resistance in C. albicans, but the biological function of Top2 in relation to drug resistance in C. albicans remains ambiguous.

In this work, we studied the effect of the TOP2 gene on drug susceptibility by disrupting both alleles of TOP 2 using the classical ura-blaster method and overexpressing the gene in the pCaEXP system in C. albicans. The results showed that deletion of TOP2 attenuated the drug susceptibility to some extent. In addition, potential action mechanisms of the TOP2 gene were investigated by measuring the uptake and efflux of drugs, and ergosterol biosynthesis. It was found that Top2 depletion introduced an increase in ergosterol content, which in turn led to attenuation of membrane fluidity and passive diffusion of drugs, thereby resulting in reduced drug susceptibility. In addition, the increased ergosterol level in a top2 null mutant was probably the result of upregulation of ERG11.

\section{METHODS}

Strains and culturing. C. albicans strain CAI4 was kindly provided by $\mathrm{Dr}$ William A. Fonzi (Department of Microbiology and Immunology, Georgetown University, Washington, DC, USA). Details of the other strains constructed during this study are listed in Supplementary Table S1 (available with the online journal).

The ura3/ura3 strains (CAI4, T1bc and $\mathrm{T} 2 \mathrm{bc}$ ) were routinely grown on YPD medium (1\% yeast extract, $2 \%$ peptone, $2 \%$ glucose) supplemented with $0.005 \%$ uridine (Sigma) at $30{ }^{\circ} \mathrm{C}$. $\mathrm{Ura}^{+}$ transformants (OET4, OET5, PECA and PET) were cultured in SC (synthetic complete) medium ( $2 \%$ glucose, $0.67 \%$ yeast nitrogen base with various amino acids as required) lacking methionine, cysteine and uridine.

Escherichia coli $\mathrm{DH} 5 \alpha$, used as a host for plasmid construction and amplification, was grown in Luria-Bertani medium with $50 \mu \mathrm{g}$ ampicillin $\mathrm{ml}^{-1}$ at $37^{\circ} \mathrm{C}$.

Drugs. The antifungal drugs used in this study were fluconazole (Pfizer); ketoconazole, miconazole, amphotericin B and caspofungin (Sigma-Aldrich); and itraconazole, voriconazole and terbinafine (Department of Organic Chemistry, Second Military Medical University, Shanghai, PR China). Dilutions of the drugs were prepared in DMSO and stored in plastic bags at $4{ }^{\circ} \mathrm{C}$ until use.

Disruption of the C. albicans TOP2 gene. Disruption of both $C$. albicans TOP2 alleles was performed using the ura-blaster approach (Fonzi \& Irwin, 1993). A 472 bp KpnI-BglII fragment containing the $3^{\prime}$ coding region of the ORF and the $3^{\prime}$ non-coding region of the TOP2 gene was amplified with oligonucleotide primers TOP2-D-F and TOP2-D-R (Supplementary Table S2 available with the online journal) and cloned into the p5921 vector upstream of the hisGURA3-hisG cassette, yielding plasmid pTU1. In the same way, a 489 bp BamHI-HindIII fragment homologous to the $5^{\prime}$ region of the TOP2 gene was amplified with primers TOP2-U-F and TOP2-U-R (Supplementary Table S2 available with the online journal) and cloned into the downstream region of the hisG-URA3-hisG cassette of pTU1 to generate the resulting TOP2 knockout plasmid named pTU2. The orientation of the inserted PCR products of the TOP2 5' and $3^{\prime}$ regions was verified at each step by PCR. In addition, DNA sequencing of plasmid pTU2 confirmed that the cloned TOP2 gene was identical to the candidate TOP2 sequence in the Candida database (CandidaDB: http://genolist.pasteur.fr/CandidaDB/). pTU2 was then linearized with $K p n \mathrm{I}$ and used in transformation of $C$. albicans CAI4 using the lithium acetate method according to Sanglard et al. (1996). The same procedure was repeated to delete the remaining functional allele of the TOP2 gene.

DNA extraction and Southern blotting. TOP2 disruption in heterozygous and homozygous strains was verified by Southern blotting. Genomic DNA was isolated from all the strains and digested with BglII. The 811 bp hybridization probe was amplified by PCR using the primers TZ-F and TZ-R (Supplementary Table S2 available with the online journal). Labelling of the DNA probe and subsequent hybridization were carried out using a random-primed digoxigenin DNA-labelling detection kit (Roche).

Reintegration of the C. albicans TOP2 gene. The sequence of the C. albicans TOP2 gene was retrieved from the annotated CandidaDB database. The plasmid pTWD containing the complete ORF of the $C$. albicans TOP2 gene was amplified from the genomic DNA of strain CAI4 by PCR and sequenced. The vector pCaEXP was digested with BamHI and PstI to enable integration of the complete ORF of TOP2 into the MET3 promoter, producing the overexpressing vector $\mathrm{pEXP}$ TOP2. After sequencing, pEXP-TOP2 was linearized at the unique StuI site and transformed into C. albicans strain T2bc using the lithium acetate method to obtain TOP2 overexpression strains, designated OET. Simultaneously, the empty pCaEXP plasmid was digested with $S t u$ I and was used to transform separately strains CAI4 and T2bc to serve as negative controls, designated PECA and PET2, respectively. Transformed cells were selected as $\mathrm{Ura}^{+}$in SC medium without methionine, cysteine or uridine (Care et al., 1999).

RNA extraction and quantitative real-time PCR. RNA was isolated from each sample using a fungal RNAout kit (Tiandz) and samples were treated with DNase (Takara) and then reverse transcribed using avian myoblastosis virus reverse transcriptase and random sixmers (Takara). Real-time RT-PCR used to quantify gene expression levels was performed subsequently using a SYBR Green real-time PCR master mix (Fermentas) following the manufacturer's recommended protocols. Fluorescence signals were monitored using a Bio-Rad system. The threshold cycle $\left(C_{\mathrm{t}}\right)$ for target mRNA was normalized with transcripts of $18 \mathrm{~S}$ rRNA and the mean value was determined. Gene expression levels were calculated from normalized $C_{\mathrm{t}}$ results, and the mean results were used to represent relative fold changes between the experimental groups. The results were obtained from three independent experiments. Statistical significance was estimated using Student's $t$-test $(P \leqslant 0.05)$. Primer pairs specific for TOP2 (RT-FWD and RT-RVE), MDR1 (MDR1-F and MDR1-R), CDR1 (CDR1-F and CDR1-R), CDR2 (CDR2-F and CDR2-R) and ERG (ERG-F and ERG-R) are listed in Supplementary Table S2 (available with the online journal).

MIC determination. Drug sensitivity testing was performed by the broth microdilution technique as described by Espinel-Ingroff et al. (1995). The MIC values of the top $2 \Delta / \Delta$ mutant towards the antifungal drugs described above were determined as optical density values at $630 \mathrm{~nm}$ using a microplate reader after $24 \mathrm{~h}$ incubation at $30{ }^{\circ} \mathrm{C}$ (see Table 3). DMSO, which was used to dissolve the antifungals in the experiments, was also used as a negative control. The results shown are typical of one determination, which was confirmed by three independent experiments.

Spot assay. To further assess the resistant genotype of top2 mutants to the drugs, the mutant strains of $C$. albicans were studied using a spot assay. The C. albicans strains were grown overnight and then suspended in medium to an $\mathrm{OD}_{600}$ of 0.1 . Serial tenfold dilutions of each strain were prepared. Five microlitres of each dilution of each Candida culture was spotted onto the appropriate medium. The 
wild-type strain (CAI4) and top2 heterozygous and homozygous mutant strains (T1bc and T2bc) were spotted onto YPD plates with uridine and the indicated antifungal agents. The TOP2 overexpression mutants (OET4 and OET5) and the control strains (PET2 and PECA) were spotted onto SC medium plates (without methionine, cysteine or uridine) with a specific antifungal compound as indicated. The differences between the strains were observed after incubation for 2 days at $30{ }^{\circ} \mathrm{C}$.

Rhodamine 6G efflux assay. C. albicans cells were grown in appropriate medium at $30{ }^{\circ} \mathrm{C}$ for $16 \mathrm{~h}$, subcultured into the same medium and incubated at $30{ }^{\circ} \mathrm{C}$ for $5 \mathrm{~h}$. Cells were then washed three times in PBS, and resuspended in PBS at an $\mathrm{OD}_{600}$ of 2.0 to exhaust their energy. Rhodamine $6 \mathrm{G}(10 \mu \mathrm{M})$ was added to the de-energized cells and incubation was carried out for a further $45 \mathrm{~min}$. The cells were washed three times and the final concentration of cells was kept at $5 \times 10^{7}$ cells ml ${ }^{-1}$. Aliquots of cells were pre-incubated at $30{ }^{\circ} \mathrm{C}$ for $5 \mathrm{~min}$, and rhodamine $6 \mathrm{G}$ efflux from the cells was then initiated by the addition of $2 \mathrm{mM}$ glucose to stimulate efflux, with continuous incubation at $30{ }^{\circ} \mathrm{C}$. At specified time points after the addition of glucose, cells were collected from duplicate assays by centrifugation, and $100 \mu \mathrm{l}$ aliquots of the cell supernatant were transferred to the wells of 96-well flat-bottomed microtitre plates (BMG Labtech). The fluorescence signal of rhodamine $6 \mathrm{G}$ in the samples was measured using a Scientific Varioskan Flash plate reader (Thermo) using excitation and emission wavelengths of 515 and $555 \mathrm{~nm}$, respectively.

Quantitative sterol analysis. C. albicans cells (strains CAI4, T2bc and OET4 in the absence or presence of $2 \mu \mathrm{g}$ fluconazole $\mathrm{ml}^{-1}$ ) incubated for $24 \mathrm{~h}$ were harvested by centrifugation at 5000 r.p.m. (Biofuge Stratos superspeed refrigerated centrifuge; Heraeus) for $5 \mathrm{~min}$, washed with PBS three times and $0.5 \mathrm{mg}$ thallus was measured separately. The cell pellets were saponified using saponification agent at $80{ }^{\circ} \mathrm{C}$ for $1 \mathrm{~h}$. Non-saponifiable lipids (ergosterols and ergosterol precursors) were extracted with petroleum ether (at 30-60 ${ }^{\circ} \mathrm{C}$ ) three times, dried at $60{ }^{\circ} \mathrm{C}$ and then dissolved in cyclohexane. Sterols were analysed by GC/high-resolution MS (GC/HRMS) using split injections with a split ratio of $10: 1$. The sterol composition was quantified based on comparing relative retention times to that of an internal standard (cholesterol). The operating conditions used for GC/HRMS were as described by Howell et al. (1990).

Passive diffusion assay. Passive diffusion of rhodamine 6G was determined by a published protocol (Mukhopadhyay et al., 2002). Briefly, Candida cells were collected and washed in PBS without glucose and then resuspended in PBS (without glucose) supplemented with $5 \mathrm{mM}$ dinitrophenol and $5 \mathrm{mM}$ 2-deoxy-D-glucose. Rhodamine 6G $(10 \mu \mathrm{M})$ was added to the de-energized cell suspension and incubated for $40 \mathrm{~min}$. The cells were pelleted and the absorbance of the supernatant was measured spectrophotometrically at $527 \mathrm{~nm}$.

Detection of fluorescence polarization. Steady-state fluorescence polarization measurements of $C$. albicans cells were carried out with the fluorescent probe 1,6-diphenyl-1,3,5-hexatriene at excitation and emission wavelengths of 360 and $426 \mathrm{~nm}$, respectively (Prasad et al., 2005).

\section{RESULTS AND DISCUSSION}

\section{Generation and identification of top $2 \Delta / \Delta$ mutants and reintegration mutants}

The role of the TOP2 gene in C. albicans was studied by deleting the two top2 alleles in strain CAI4. The ura-blaster strategy is shown in Supplementary Fig. S1(a) (available with the online journal). The strains produced in this process are listed in Supplementary Table S1. Southern blotting showed that both top2 alleles were disrupted successfully (Supplementary Fig. S1b available with the online journal).

The TOP2 ORF was placed under the control of the MET3 promoter in pCaEXP and transformed into the top 2 null mutant (strain T2bc). Relative quantification by real-time RT-PCR was used to confirm overexpression of the TOP2 gene in OET (T2bc harbouring pEXP-TOP2) compared with its expression in PECA (CAI4 harbouring pCaEXP). The TOP2 expression level in OET4 and OET5 was 15.7fold and 8.7-fold higher, respectively, than that in PECA (Supplementary Fig. S2 available with the online journal).

\section{top $2 \Delta / \Delta$ mutant shows a reduction in drug susceptibility}

To identify the role of the TOP2 gene in drug susceptibility, the phenotype of the parental strain (CAI4) and the top 2 null mutant (T2bc) were studied. No significant difference in the growth rate of CAI4 and T2bc was observed (Supplementary Fig. S3, available with the online journal). As shown in Table 1, T2bc had a higher MIC value and was less susceptible to azoles compared with CAI4. The MIC value of $\mathrm{T} 2 \mathrm{bc}$ increased 16-fold in the case of fluconazole and ketoconazole, eightfold for voriconazole, and fourfold for miconazole and itraconazole, compared with that for CAI4.

Spot assay analysis also showed that the null mutant T2bc was less susceptible to fluconazole, miconazole, ketoconazole, itraconazole and voriconazole relative to native CAI4 (Fig. 1a), which was consistent with the MIC results, whilst the TOP2 overexpression strains (OET4 and OET5) became more susceptible (Fig. 1b). To see whether the decreased susceptibility of C. albicans as a result of Top2

Table 1. MIC values of the wild-type strain CAI4 and top2 mutants T1bc and T2bc, tested by the broth microdilution method

\begin{tabular}{|lccccc|}
\hline \multirow{2}{*}{ Strain } & \multicolumn{5}{c|}{ MIC $\left(\boldsymbol{\mu g ~ \mathbf { ~ m } ^ { - \mathbf { 1 } } )}\right.$} \\
\cline { 2 - 6 } & Fluconazole & Miconazole & Ketoconazole & Itraconazole & Voriconazole \\
\hline CAI4 & 0.250 & 0.250 & 0.015 & 0.050 & 0.001 \\
T1bc & 2.000 & 0.500 & 0.125 & 0.100 & 0.001 \\
T2bc & 4.000 & 1.000 & 0.250 & 0.200 & 0.008 \\
\hline
\end{tabular}


deprivation was limited to azoles, the spot assay was also performed with amphotericin B, terbinafine and caspofungin. T2bc also displayed attenuated susceptibility to these drugs, indicating that the TOP2 expression levels did not only affect azole susceptibility.

\section{TOP2 gene does not influence efflux activity}

Resistance of $C$. albicans to azole antifungals is often caused by increased expression of genes encoding multidrug efflux pumps, such as MDR1, CDR1 and CDR2. Real-time RTPCR determination of the expression levels of $M D R 1$, $C D R 1$ and $C D R 2$ revealed that there was no significant alteration between the wild-type (CAI4) and the top2 mutant strains (T2bc and OET4) (see Supplementary Fig. S4 available with the online journal).

In addition, the function of Cdrlp was studied by determining rhodamine $6 \mathrm{G}$ efflux. The effect of the expression of the TOP2 gene on energy-dependent drug efflux was quantified by comparably pre-loading the top $2 \Delta / \Delta$ mutant (T2bc) and wild-type strain (CAI4) with the pump substrate rhodamine 6G. As shown in Supplementary Fig. S5 (available with the online journal), the function of efflux transporters was weak without a glucose supply, but was enhanced significantly after the addition of glucose to both strains, although the difference between them was negligible. In conclusion, the study on drug transporter genes and the function of Cdrlp indicated that the resistant phenotype of the top $2 \Delta / \Delta$ null mutant was not associated with drug efflux activity.

\section{TOP2 gene affects ergosterol biosynthesis in C. albicans}

It is known that abnormalities of ergosterol biosynthesis are another cause of decreased drug availability in cells; therefore, the amount of intracellular ergosterol present in top2 mutants (T2bc and OET4) and the isogenic wild-type strain (CAI4) was quantified by GC/HRMS. The results in
Table 2 showed that the ability of the top $2 \Delta / \Delta$ null mutant (T2bc) to biosynthesize ergosterol was enhanced significantly, with the greatest amount of ergosterol $(81.80 \%)$, whilst there was a reproducible decrease in ergosterol in OET4 $(44.50 \%)$. Simultaneously, when treated with $2 \mu \mathrm{g}$ fluconazole $\mathrm{ml}^{-1}$, the strains accumulated large amounts of $14 \alpha$-methylated sterol in the form of 24,25 -dihydrolanosterol. The ergosterol percentage of T2bc was markedly reduced from 81.80 to $16.36 \%$. In strains CAI4 and OET4, ergosterol levels decreased sharply to an undetectable level. This result is consistent with upregulation of the sterol biosynthetic pathway.

\section{ERG11 gene expression is upregulated in the top $2 \Delta / \Delta$ mutant}

Determination of sterol composition by GC/HRMS analysis showed that expression of the TOP2 gene altered the drug sensitivity of $C$. albicans by contributing to ergosterol biosynthesis. Accordingly, gene expression levels of ERG2, ERG3, ERG11, ERG24 and ERG25 were measured. As expected, the expression level of the ERG11 gene was increased in T2bc by more than twofold compared with CAI4. In contrast, overexpression of TOP2 resulted in downregulation of ERG11 in OET4 by approximately twofold compared with CAI4 (Fig. 2). Statistical analysis showed that there was a significant difference in ERG11 gene expression among the experimental groups $(P \leqslant 0.05)$. No obvious differences in the expression of ERG2, ERG3, ERG24 and ERG25 were observed among the experimental groups.

\section{Top2 depletion leads to a decrease in diffusion of drugs and membrane fluidity}

It is known that there is an intricate relationship between membrane fluidity and ergosterol content. In view of the increased ergosterol level in the top $2 \Delta / \Delta$ null mutant, passive diffusion and membrane fluidity were explored.

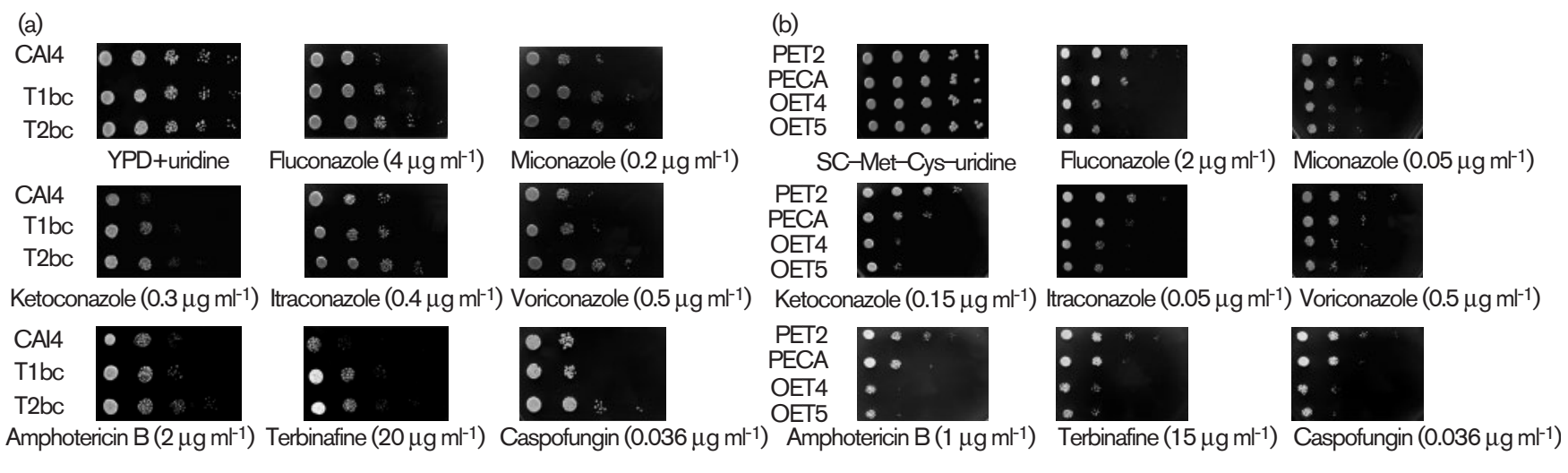

Fig. 1. Drug resistance profiles of C. albicans wild-type and top2 mutant cells determined by spot assay as described in Methods. The names of the strains are indicated on the left. 
Table 2. Quantification of sterol content in C. albicans strains CAI4, T2bc and OET4 with or without fluconazole by GC/HRMS

\begin{tabular}{|c|c|c|c|c|c|}
\hline Treatment & Strain & Squalene & Ergosterol & Lanosterol & $\begin{array}{c}24,25- \\
\text { Dihydrolanosterol }\end{array}$ \\
\hline \multirow[t]{3}{*}{ Control $\dagger$} & CAI4 & $5.48 \pm 0.86$ & $64.49 \pm 4.55$ & $30.01 \pm 1.86$ & ND \\
\hline & $\mathrm{T} 2 \mathrm{bc}$ & $1.94 \pm 0.76$ & $81.80 \pm 1.01$ & $16.26 \pm 1.78$ & ND \\
\hline & OET4 & $\mathrm{ND}$ & $44.50 \pm 2.76$ & $55.50 \pm 2.76$ & $\mathrm{ND}$ \\
\hline \multirow{2}{*}{ Fluconazole $\left(2 \mu \mathrm{g} \mathrm{ml}^{-1}\right)$} & $\mathrm{T} 2 \mathrm{bc}$ & $\mathrm{ND}$ & $16.36 \pm 4.65$ & $26.63 \pm 5.82$ & $57.00 \pm 1.17$ \\
\hline & OET4 & $\mathrm{ND}$ & $\mathrm{ND}$ & $38.97 \pm 2.22$ & $61.03 \pm 2.22$ \\
\hline
\end{tabular}

ND, Not detected.

${ }^{*}$ All samples were taken from triple independent experiments and are represented as mean \pm SD.

$\dagger$ Control (in the absence of fluconazole).

Fluorescence polarization measurements revealed that TOP2 disruption led to a $36.5 \%$ decrease in membrane fluidity, resulting in decreased passive diffusion of rhodamine $6 \mathrm{G}$ by $30.3 \%$ (Table 3 ). These results implied that the intake of rhodamine $6 \mathrm{G}$ was less in the top $2 \Delta / \Delta$ mutant than in the wild type. Taken together, the decreased passive diffusion of rhodamine $6 \mathrm{G}$ by the top $2 \Delta / \Delta$ mutant indicated that the top $2 \Delta / \Delta$ mutant membrane was less permeable, a property that could explain the reduced sensitivity to the drugs.

As overexpression of drug efflux pump-encoding genes is the predominant mechanism responsible for drug resistance, we first hypothesized that the observed phenotype of top2 mutants in drug susceptibility might be the result of changes in drug efflux. However, TOP2 disruption was not able to modify the expression levels of the multidrug transporter genes. Thus, the effect of TOP2 expression on drug sensitivity appeared to be independent of the efflux pump proteins. However, the ergosterol content of the top 2 null mutation strain was higher than that of the parental strain, which could account for the lowered membrane fluidity and passive diffusion of drugs observed. Our results demonstrate for the first time that mutations in the TOP2 gene could play a role in the mechanism of Candida

Table 3. Measurements of membrane fluidity and passive diffusion of top2 mutants and the wild-type strain

\begin{tabular}{|lcc|}
\hline Strain & P value $^{\star}$ & OD $_{527}{ }^{\dagger}$ \\
\hline CAI4 & $0.202 \pm 0.013$ & $0.344 \pm 0.022$ \\
T2bc & $0.275 \pm 0.012$ & $0.449 \pm 0.023$ \\
OET4 & $0.145 \pm 0.008$ & $0.256 \pm 0.013$ \\
\hline
\end{tabular}

*The fluorescence polarization $(\mathrm{P})$ values of cells. $\mathrm{P}$ values are the means \pm SD of three independent experiments.

$\dagger$ Extracellular rhodamine $6 \mathrm{G}$ concentrations. The values are represented as mean \pm SD of three sets of experiments. resistance to antifungal drugs. TOP2 expression appeared to have a relationship with ERG11 expression, leading to the question of why and how TOP2 negatively affects ERG11 expression. An attempt to further understand the potentially insidious functions and mechanisms of action of Top2 is under way in our laboratory.

It has now been established that the Top2 protein maintains a favourable nucleic acid topology for replication, transcription, control of recombination and chromosome segregation, which are indispensable for normal growth of the organism (Nitiss, 1998; Cline \& Hanawalt, 2006). However, C. albicans (CAI4) with both TOP2 alleles disrupted was able to survive, and TOP2 deletion did not affect the growth rate of the strains. Therefore, the TOP2 gene does not seem

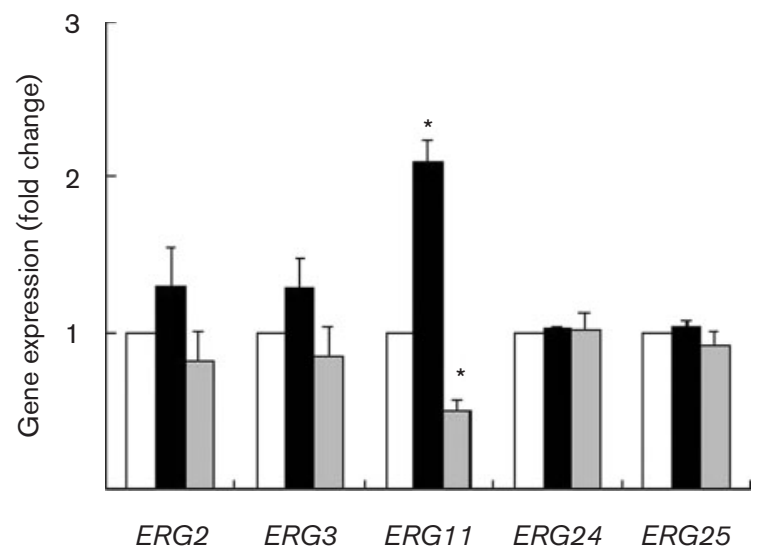

Fig. 2. Expression levels of $C$. albicans $E R G$ genes in strains CAl4 (white bars), T2bc (black bars) and OET4 (grey bars) determined by RT-PCR. RNA levels (normalized to $18 \mathrm{~S}$ rRNA levels) are represented as fold increase or decrease relative to the level for the control strain (CAI4). Error bars represent SEM. Asterisks denote statistical significance by Student's $t$-test $(P \leqslant 0.05)$. 
essential for growth in C. albicans, probably because Top2 could be substituted by the roles of some functional complementation genes. The current view assumes that supercoiling generated by helix unwinding is mainly relaxed by type IB enzymes (Schvartzman \& Stasiak, 2004). The results of Kim \& Wang (1989) showed that synthesis of short DNA chains can initiate in the absence of both topoisomerases in S. cerevisiae, but their further elongation requires at least one of the two topoisomerases. Inactivation of Top2 does not significantly alter the patterns of nascent DNA chain synthesis (Kim \& Wang, 1989). Yeast cells can replicate DNA in top1 or top2 mutants, but not when both Top1 and Top2 functions are defective, suggesting that TOP1 might play an important role in assisting chromosome replication in the absence of Top2. Although extensive biochemical and structural studies in the past have determined the cellular roles of the enzymes, their exact roles in the process of DNA synthesis within the eukaryotic chromatin context remain unclear. Moreover, Top2 may possess other critical and unexpected biological functions in C. albicans, and this needs further study.

In summary, the results of the present study demonstrated that mutations in the TOP2 gene correlated with the alteration of drug susceptibility in C. albicans. It is possible that the increased transcription level of ERG11 in the TOP2-deleted strain augmented ergosterol biosynthesis, thus eliciting the decreased membrane fluidity and passive diffusion of drugs, leading ultimately to reduced susceptibility to the antifungal drugs. The influence of Top2 on the drug susceptibility of Candida suggests that Top2 might be another novel determinant of multidrug resistance.

\section{ACKNOWLEDGEMENTS}

This work was supported by the National Basic Research Project (2005CB523105), National Natural Science Foundation of China (30500628, 30825041 and 30630071), Chinese National Programs for High Technology Research and Development (2007AA02Z187) and Shanghai Young Scientist Development Foundation (07QH14019 and 2007CG51). The authors thank William A. Fonzi (Department of Microbiology and Immunology, Georgetown University, Washington, DC, USA) for kindly providing C. albicans strain CAI4.

\section{REFERENCES}

Akins, R. A. (2005). An update on antifungal targets and mechanisms of resistance in Candida albicans. Med Mycol 43, 285-318.

Albertson, G. D., Niimi, M., Cannon, R. D. \& Jenkinson, H. F. (1996). Multiple efflux mechanisms are involved in Candida albicans fluconazole resistance. Antimicrob Agents Chemother 40, 2835-2841.

Anderson, J. B. (2005). Evolution of antifungal-drug resistance: mechanisms and pathogen fitness. Nat Rev Microbiol 3, 547-556.

Care, R. S., Trevethick, J., Binley, K. M. \& Sudbery, P. E. (1999). The MET3 promoter: a new tool for Candida albicans molecular genetics. Mol Microbiol 34, 792-798.

Cline, S. D. \& Hanawalt, P. C. (2006). Topoisomerase deficiencies subtly enhance global genomic repair of ultraviolet-induced DNA damage in Saccharomyces cerevisiae. DNA Repair (Amst) 5, 611-617.
Dassanayake, R. S., Ellepola, A. N., Samaranayake, Y. H. \& Samaranayak, L. P. (2002). Molecular heterogeneity of fluconazoleresistant and -susceptible oral Candida albicans isolates within a single geographic locale. APMIS 110, 315-324.

Espinel-Ingroff, A., Rodríguez-Tudela, J. L. \& Martínez-Suárez, J. V. (1995). Comparison of two alternative microdilution procedures with the National Committee for Clinical Laboratory Standards reference macrodilution method M27-P for in vitro testing of fluconazole-resistant and -susceptible isolates of Candida albicans. J Clin Microbiol 33, 3154.

Fonzi, W. A. \& Irwin, M. Y. (1993). Isogenic strain construction and gene mapping in Candida albicans. Genetics 134, 717-728.

Ghannoum, M. A. \& Rice, L. B. (1999). Antifungal agents: mode of action, mechanism of resistance and correlation of these mechanisms with bacterial resistance. Clin Microbiol Rev 12, 501-517.

Howell, S. A., Mallet, A. I. \& Noble, W. C. (1990). A comparison of the sterol content of multiple isolates of the Candida albicans Darlington strain with other clinically azole sensitive and resistant strains. J Appl Bacteriol 69, 692-696.

Keller, B. A., Patel, S. \& Fisher, L. M. (1997). Molecular cloning and expression of the Candida albicans TOP2 gene allows study of fungal DNA topoisomerase II inhibitors in yeast. Biochem J 324, 329-339.

Kim, R. A. \& Wang, J. C. (1989). Function of DNA topoisomerases as replication swivels in Saccharomyces cerevisiae. J Mol Biol 208, 257-267.

Kohli, A., Smriti, Mukhopadhyay, K., Rattan, A. \& Prasad, R. (2002). In vitro low-level resistance to azoles in Candida albicans is associated with changes in membrane lipid fluidity and asymmetry. Antimicrob Agents Chemother 46, 1046-1052.

Loffler, J., Einsele, H., Hebart, H., Schumacher, U., Hrastnik, C. \& Daum, G. (2000). Phospholipid and sterol analysis of plasma membranes of azole-resistant Candida albicans strains. FEMS Microbiol Lett 185, 59-63.

Morschhauser, J. (2002). The genetic basis of fluconazole resistance development in Candida albicans. Biochim Biophys Acta 1587, 240-248.

Mukhopadhyay, K., Kohli, A \& Prasad, R. (2002). Drug susceptibilities of yeast cells are affected by membrane lipid composition. Antimicrob Agents Chemother 46, 3695-3705.

Nitiss, J. L. (1998). Investigating the biological functions of DNA topoisomerases in eukaryotic cells. Biochim Biophys Acta 1400, 63-81.

Pappas, P. G., Rex, J. H., Lee, J., Hamill, R. J., Larsen, R. A., Powderly, W., Kauffman, C. A., Hyslop, N., Mangino, J. E. \& other authors (2003). A prospective observational study of candidemia: epidemiology, therapy, and influences on mortality in hospitalized adult and pediatric patients. Clin Infect Dis 37, 634-643.

Perea, S. \& Patterson, T. F. (2002). Antifungal resistance in pathogenic fungi. Clin Infect Dis 35, 1073-1080.

Perea, S., López-Ribot, J. L., Kirkpatrick, W. R., McAtee, R. K., Santillán, R. A., Martínez, M., Calabrese, D., Sanglard, D. \& Patterson, T. F. (2001). Prevalence of molecular mechanisms of resistance to azole antifungal agents in Candida albicans strains displaying high-level fluconazole resistance isolated from human immunodeficiency virusinfected patients. Antimicrob Agents Chemother 45, 2676-2684.

Prasad, T., Saini, P., Gaur, N. A., Vishwakarma, R. A., Khan, L. A., Haq, Q. M. \& Prasad, R. (2005). Functional analysis of CaIPT1, a sphingolipid biosynthetic gene involved in multidrug resistance and morphogenesis of Candida albicans. Antimicrob Agents Chemother 49, 3442-3452.

Sanglard, D., Ischer, F., Monod, M. \& Bille, J. (1996). Susceptibilities of Candida albicans multidrug transporter mutants to various antifungal agents and other metabolic inhibitors. Antimicrob Agents Chemother 40, 2300-2305.

Schvartzman, J. B. \& Stasiak, A. (2004). A topological view of the replicon. EMBO Rep 5, 256-261. 
Shao, P. L., Huang, L. M. \& Hsueh, P. R. (2007). Recent advances and challenges in the treatment of invasive fungal infections. Int $J$ Antimicrob Agents 30, 487-495.

Silver, P. M., Oliver, B. G. \& White, T. C. (2004). Role of Candida albicans transcription factor Upc2p in drug resistance and sterol metabolism. Eukaryot Cell 3, 1391-1397.

Umeyama, T., Nagai, Y., Niimi, M. \& Uehara, Y. (2002). Construction of FLAG tagging vectors for Candida albicans. Yeast 19, 611618.

Wang, J. C. (2002). Cellular roles of DNA topoisomerases: a molecular perspective. Nat Rev Mol Cell Biol 3, 430-440.
White, T. C., Holleman, S., Dy, F., Mirels, L. F. \& Stevens, D. A. (2002). Resistance mechanisms in clinical isolates of Candida albicans. Antimicrob Agents Chemother 46, 1704-1713.

Wisplinghoff, H., Bischoff, T., Tallent, S. M., Seifert, H., Wenzel, R. P. \& Edmond, M. B. (2004). Nosocomial bloodstream infections in US hospitals: analysis of 24,179 cases from a prospective nationwide surveillance study. Clin Infect Dis 39, 309-317.

Zini, N., Martelli, A. M., Sabatelli, P., Santi, S., Negri, C., Astaldi Ricotti, G. C. \& Maraldi, N. M. (1992). The $180-\mathrm{kDa}$ isoform of topoisomerase II is localized in the nucleolus and belongs to the structural elements of the nucleolar remnant. Exp Cell Res 200, 460-466. 